\title{
BMJ Open Readmission and processes of care across weekend and weekday hospitalisation for acute myocardial infarction, heart failure or stroke: an observational study of the National Readmission Database
}

Glen Philip Martin, ${ }^{\oplus 1}$ Chun Shing Kwok, ${ }^{2}$ Harriette Gillian Christine Van Spall, ${ }^{3}$ Annabelle Santos Volgman, ${ }^{4}$ Erin Michos, ${ }^{5}$ Purvi Parwani, ${ }^{6}$ Chadi Alraies, ${ }^{7}$ Ritu Thamman, ${ }^{8}$ Evangelos Kontopantelis, ${ }^{1}$ Mamas Mamas ${ }^{2}$

To cite: Martin GP, Kwok CS Van Spall HGC, et al. Readmission and processes of care across weekend and weekday hospitalisation for acute myocardial infarction, heart failure or stroke: an observational study of the National Readmission Database. BMJ Open 2019;9:e029667. doi:10.1136/ bmjopen-2019-029667

- Prepublication history and additional material for this paper are available online. To view these files, please visit the journal online (http://dx.doi org/10.1136/bmjopen-2019029667).

Received 26 February 2019

Revised 30 June 2019

Accepted 03 July 2019

A Check for updates

(C) Author(s) (or their employer(s)) 2019. Re-use permitted under CC BY-NC. No commercial re-use. See rights and permissions. Published by BMJ.

For numbered affiliations see end of article.

Correspondence to

Prof Mamas Mamas;

mamasmamas1@yahoo.co.uk

\section{ABSTRACT}

Objectives Variation in hospital resource allocations across weekdays and weekends have led to studies of the 'weekend effect' for ST elevation myocardial infarction (STEMI), non-ST elevation myocardial infarction (NSTEMI), heart failure (HF) and stroke. However, few studies have explored the 'weekend effect' on unplanned readmission. We aimed to investigate 30 -day unplanned readmissions and processes of care across weekend and weekday hospitalisations for STEMI, NSTEMI, HF and stroke.

Design We grouped hospitalisations for STEMI, NSTEMI, HF or stroke into weekday or weekend admissions. Multivariable adjusted ORs for binary outcomes across weekend versus weekday (reference) groups were estimated using logistic regression.

Setting We included all non-elective hospitalisations for STEMI, NSTEMI, HF or stroke, which were recorded in the US Nationwide Readmissions Database between 2010 and 2014.

Participants The analysis sample included 659906 hospitalisations for STEMI, 1420600 hospitalisations for NSTEMI, 3027699 hospitalisations for HF, and 2574168 hospitalisations for stroke.

Main outcome measures The primary outcome was unplanned 30-day readmission. As secondary outcomes, we considered length of stay and the following processes of care: coronary angiography, primary percutaneous coronary intervention, coronary artery bypass graft, thrombolysis, brain scan/imaging, thrombectomy, echocardiography and cardiac resynchronisation therapy/ implantable cardioverter-defibrillator.

Results Unplanned 30-day readmission rates were $11.0 \%, 15.1 \%, 23.0 \%$ and $10.9 \%$ for STEMI, NSTEMI, HF and stroke, respectively. Weekend hospitalisations for HF were associated with a statistically significant but modest increase in 30-day readmissions (OR of $1.045,95 \% \mathrm{Cl}$ 1.033 to 1.058). Weekend hospitalisation for STEMI, NSTEMI or stroke was not associated with increased risk of 30-day readmission.
Strengths and limitations of this study

A study exploring the effect of weekend hospitalisation for ST elevation myocardial infarction (STEMI), non-ST elevation myocardial infarction (NSTEMI), heart failure (HF) and stroke on unplanned 30-day readmission.

- Study included 659906 hospitalisations for STEMI, 1420600 hospitalisations for NSTEMI, 3027699 hospitalisations for HF and 2574168 hospitalisations for stroke recorded in the National Readmissions Database (NRD) of the USA.

- The annualised nature of the NRD means that we were not able to track patients between years, thereby limiting investigation to short-term readmission.

- We could not investigate diurnal variation in outcomes or by individual day of hospitalisation.

Conclusion There was no clinically meaningful evidence against the supposition that weekend and weekday hospitalisations have the same 30-day unplanned readmissions. Thirty-day readmission rates were high, especially for HF, which has implications for service provision. Strategies to reduce readmission rates should be explored, regardless of day of hospitalisation.

\section{INTRODUCTION}

Cardiovascular disease is one of the leading causes of mortality and morbidity worldwide, ${ }^{1}$ with coronary heart disease and stroke being the primary causes of cardiovascular-related mortality. ${ }^{2}$ Timely diagnosis and treatment is key to improving prognosis following acute myocardial infarction (MI), heart failure (HF) and stroke/transient ischaemic attack (TIA). However, staffing levels, resource allocation and service provision are known to vary 
between weekdays and weekends, ${ }^{3}$ which could impact on the processes of care for these diagnoses. Such a hypothesis has resulted in numerous studies of the so-called 'weekend effect' that aim to explore associations between weekend hospitalisation and clinical outcomes. ${ }^{4-7}$ Nevertheless, the existing evidence base is largely inconsistent; one explanation is that the effect of a weekend hospitalisation on clinical outcomes is likely to depend on recommended treatment processes and on service provision across hospitals.

For example, current guidelines recommend early diagnosis and treatment for ST elevation myocardial infarction (STEMI) with primary percutaneous coronary intervention (PCI). Among its many benefits, primary PCI is a $24 / 7$ service, hence there is arguably limited potential of a weekend effect in this cohort. Similarly, revascularisation for non-ST elevation myocardial infarction (NSTEMI) is recommended within 72 hours $^{8-11}$ and emerging direct transfer protocols ${ }^{12}$ could again limit potential of a weekend effect. In contrast, while many acute care hospitals provide routine care for $\mathrm{HF}$ on a weekday, there are differences in staffing levels at a weekend, ${ }^{13}$ suggesting that specialists might not see patients hospitalised for HF as quickly. Similarly, for stroke/TIA, thrombolysis or thrombectomy are immediately indicated for subgroups of patients, but availability of these resources locally could cause heterogeneity in the receipt of these processes of care by day of the week. ${ }^{14}$

There have been numerous studies investigating in-hospital outcomes across weekday/weekend hospitalisations for STEMI, NSTEMI, HF and stroke/TIA. ${ }^{14-24}$ However, there is a paucity of data surrounding unplanned readmissions following weekend hospitalisations for these diagnoses, with the majority of prior evidence focusing on mortality. Studying the effect of weekend hospitalisation on unplanned 30-day readmissions is important since they have implications on resource utilisation and indicators of care quality, and they can lead to financial penalties. To the best of our knowledge, no previous study has simultaneously contrasted the readmission rates and processes of care for these primary diagnoses across weekend and weekday hospitalisation groups.

Therefore, this study aimed to use a national readmission database of the USA to explore the weekend effect with respect to 30-day unplanned readmission and processes of care in patients admitted for STEMI, NSTEMI, HF or stroke/TIA.

\section{METHODS}

\section{Cohort description}

This was a retrospective cohort analysis of the 2010-2014 Agency for Healthcare Research and Quality's Nationwide Readmissions Database (NRD), which is a subset of the Healthcare Cost and Utilization Project (HCUP). NRD is a database of hospital inpatient stays for patients of all ages and for all payers in the USA. The data are drawn from 21 states that are geographically dispersed and accounts for approximately $49 \%$ of total US resident population and hospitalisations. ${ }^{25}$ The NRD includes a weighting (provided by HCUP) that are applied to account for the complex survey design and produce national estimates. Readmissions for an individual patient can be identified within a given calendar year using a deidentified unique patient linkage number.

\section{Patient and public involvement}

We did not directly include patients/public in this study.

\section{Ethics approval}

As per the Agency for Healthcare Research and Quality, this study was except from review by an institutional review board because data are publicly available and deidentified.

\section{Study design and setting}

Index hospitalisations were identified between January and November within each calendar year (with hospitalisations in December of each year excluded to allow identification of 30-day readmission rates for all patients, since NRD is annualised and cannot track patients between years). During this period, index hospitalisations were defined as any non-elective admission with a primary International Statistical Classification of Diseases and Related Health Problems (ICD)-9 diagnosis code of: (1) STEMI (ICD-9 codes: 410.0*, 410.1*, 410.2*, $410.3^{*}, 410.4^{*}, 410.5^{*}, 410.6^{*}, 410.8^{*}$ and $410.9^{*}$, each excluding those coded as having a subsequent episode of care), (2) NSTEMI (ICD-9 codes: 410.7*, excluding those coded as having a subsequent episode of care), 3) HF (ICD-9 codes: 402.01, 402.11, 402.91, 404.01, 404.03, $404.11,404.13,404.91,404.93$ and $428.00-428.91)^{26}$ or (4) stroke/TIA (ICD-9 codes: 430, 431, 433.*1, 434.*1, 435.* and 436). ${ }^{27-29}$ Across multiple hospitalisations, a patient could appear in more than one diagnosis group. For example, if a patient was first admitted for STEMI and was then readmitted for HF within 30-days, then the admission for HF acts as both a readmission event (for the initial STEMI hospitalisation) and also as a new index hospitalisation (for HF) to allow us to explore subsequent readmission events. Index hospitalisations were excluded if: (1) the patient was aged younger than 18 years, (2) the patient died during the index hospitalisation, (3) there was no information on the length of stay (LOS), (4) we could not determine whether the hospitalisation was at a weekday or weekend and/or (5) the hospitalisation was coded elective. Hospital transfers or same-day stays, were excluded (identified using the variables 'SAMEDAYEVENT' and 'REHABTRANSFER' in the NRD).

We grouped patients into those admitted on a weekday (Monday-Friday) or at a weekend (Saturday-Sunday), using the day of the index hospitalisation (determined using the 'AWEEKEND' variable in NRD). All analyses were stratified by the primary diagnosis category of the index hospitalisation (ie, STEMI, NSTEMI, HF or stroke/ TIA). 


\section{Patient and hospital characteristics}

Baseline patient characteristics such as age, discharge destination, sex, primary expected payer and median household income were extracted from the NRD. Additionally, we used ICD-9 codes to define several comorbidities including previous MI, previous PCI, previous coronary artery bypass graft (CABG), previous stroke/ TIA and smoking status; the codes used to define these comorbidities are given in online supplementary table 1. The following additional comorbidities are directly recorded in NRD: alcohol abuse, deficiency anaemias, rheumatoid arthritis, chronic blood loss, congestive HF, chronic pulmonary disease, coagulopathy, depression, diabetes, drug abuse, hypertension, hypothyroidism, liver disease, lymphoma, fluid and electrolyte disorders, metastatic cancer, other neurological disorders, obesity, paralysis, peripheral vascular disorders, psychoses, pulmonary circulation disorders, renal failure, solid tumour, peptic ulcer disease, valvular disease and weight loss. Hospital-level variables included bed size, rural/urban location and teaching status.

\section{Outcome measures}

All outcomes were obtained directly from NRD. We defined the primary outcome to be any unplanned (ie, non-elective) readmission occurring within the first 30 days of discharge from the index hospitalisation. If an index hospitalisation had more than one readmission within 30 days, we only included the first readmission. We determined the causes of readmission using the first diagnoses recorded using the Clinical Classification Software codes (online supplementary table 2).

As secondary outcomes, we considered length of stay (LOS) of the index hospitalisation and the following processes of care, each measured during the index hospitalisation (see online supplementary table 1 for the ICD-9 codes): coronary angiography, PCI, CABG, thrombolysis, thrombectomy, echocardiography, brain scan/imaging, and cardiac resynchronization therapy (CRT)/ implantable cardioverter-defibrillator.

\section{Statistical analysis}

For descriptive analysis, continuous variables were presented as means with SD or median and IQR, while categorical variables were presented as frequencies of occurrence. Logistic regression was used to estimate ORs for 30-day readmission across weekend versus weekday (reference) hospitalisations, where an OR $>1$ indicates weekend hospitalisation increased odds of 30-day readmission. The secondary outcomes were summarised across weekend/weekday hospitalisation groups using frequencies and percentages, and multivariable adjusted ORs were calculated. All multivariable adjusted ORs were adjusted for all of the variables listed in the 'Patient Characteristics', 'Comorbidities' and 'Hospital Characteristics' sections of table 1 . Finally, the effect of weekend hospitalisation on LOS was analysed by fitting a Cox proportional hazard model with adjustment for the aforementioned variables to obtain adjusted HRs (where a $\mathrm{HR}<1$ indicates increased risk of longer LOS, since the event here is time-to-discharge).

All analyses were performed using SAS V.9.4 (SAS Institute, Cary, NC, USA). We followed the Agency for Healthcare Research and Quality's recommendations of applying survey estimation weights to account for the complex survey design of the NRD (eg, using SAS software's PROC commands such as SURVEYLOGISTIC). Subgroup analyses were conducted using relevant domain statements within the SAS software's survey commands. All reported sample sizes, summary statistics, coefficients and confidence intervals are those obtained from the survey estimation procedures.

\section{RESULTS}

A total of 7682373 index hospitalisations were included in this analysis after applying the exclusion criteria; a flow diagram of the inclusion/exclusion criteria is given in figure 1. Of the total index hospitalisations, 659906 had a primary diagnosis of STEMI, 1420600 had a primary diagnosis of NSTEMI, 3027699 had a primary diagnosis of HF and 2574168 had a primary diagnosis of stroke/ TIA. table 1 presents the baseline characteristics (as recorded within the index hospitalisation) across each primary diagnosis group and across weekend/weekday hospitalisations.

\section{Unplanned 30-day readmissions}

Overall, there were 1263620 unplanned readmissions within 30 days (16.4\%). The 30-day unplanned readmission rates for each primary diagnosis across weekend and weekday hospitalisations are given in table 1 . Readmission rates were highest for HF $(23.6 \%$ weekend and $22.8 \%$ weekday), but they have visually decreased through time for all primary diagnoses (figure 2). After multivariable adjustment, there was no significant difference in 30-day readmission rates between weekend and weekday hospitalisations for STEMI, NSTEMI or stroke/TIA (table 2). Although weekend hospitalisations for HF had a statistically significant increase in odds of unplanned 30-day readmission compared with those admitted for HF during the week, the effect size was modest (OR of $1.045,95 \%$ CI 1.033 to 1.058). These findings remained consistent through time, with the exception of HF in 2012 where there was no significant difference between weekend and weekday hospitalisations (figure 3).

Unsurprisingly, across the weekend hospitalisation group and the weekday hospitalisation group, the main cause of readmission was related to the primary diagnosis of the index hospitalisation (online supplementary figure 1 and supplementary figure 2). For example, for those with an index HF hospitalisation, the most common cause of readmission within 30 days was recurrent HF (and similarly for STEMI, NSTEMI and stroke/ TIA). 


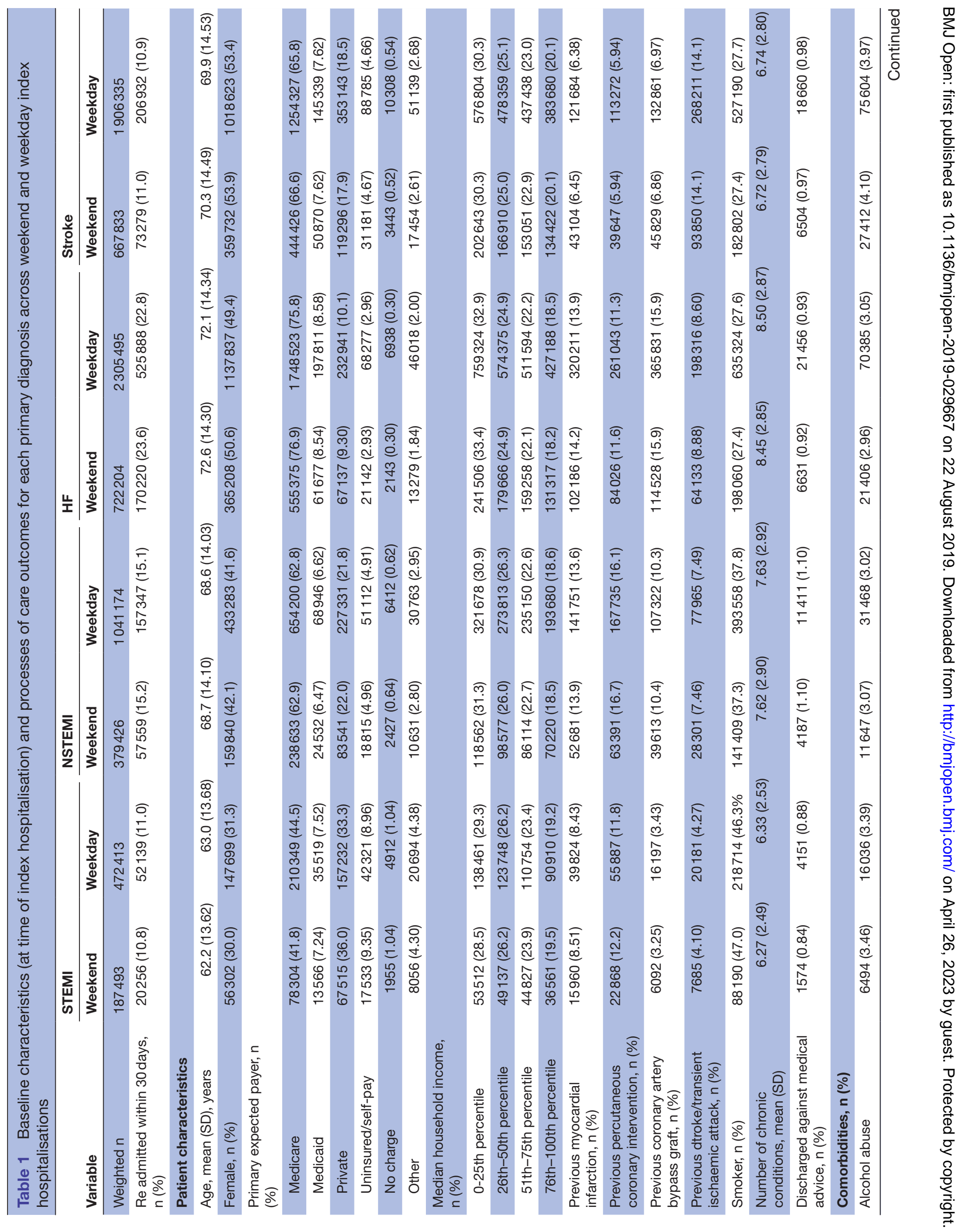


कำ

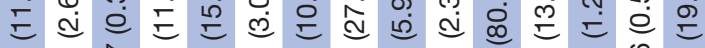
त) 范

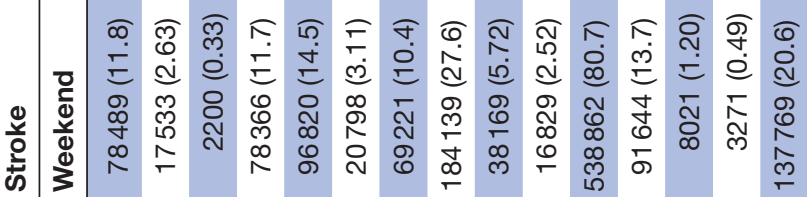

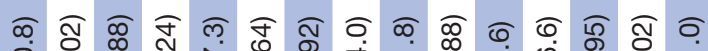
क्र

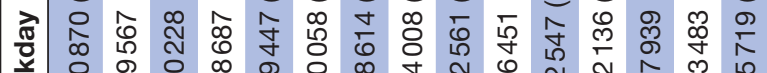
离

สุ ब চ

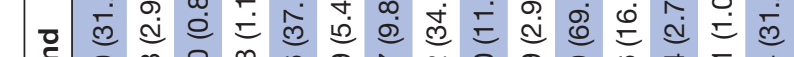

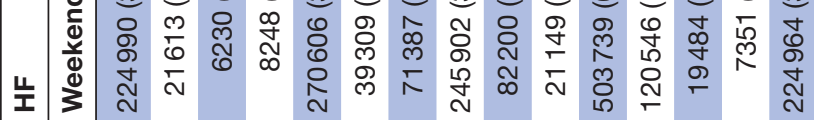

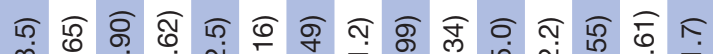

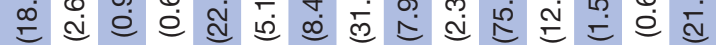

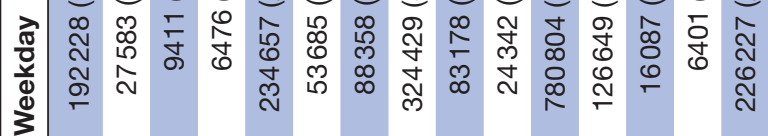

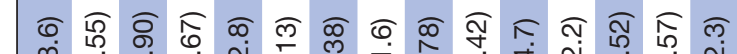

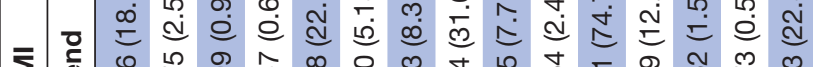

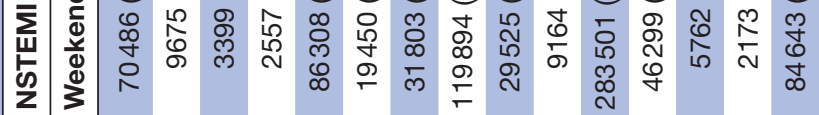

कूष

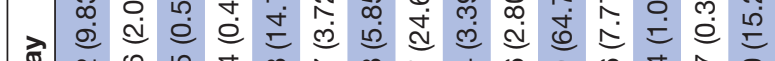

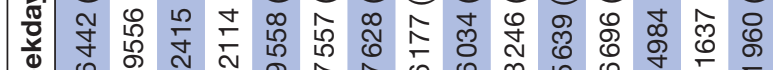
\.

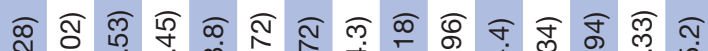
× $\frac{1}{0}$ d

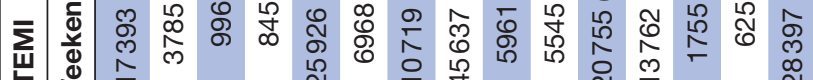

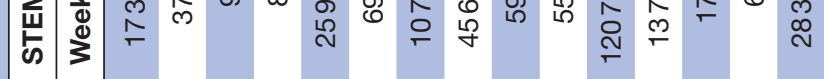

๙ิ ᄃ

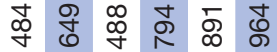
ก

๙ ¿ व లి

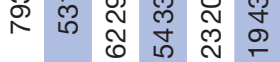

สู $\infty$

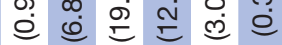

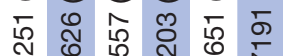
స

ठิ

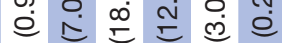
ก) लొ ๑ ก

สู สิ กิ กิ

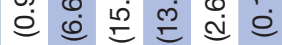
ก $\simeq$ ก เి

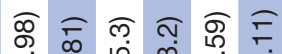
๑ న 饮

হ

เึิ สิ

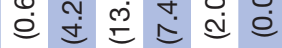
ㄸ ल न

ชิ ণ্ণ 당 ¿ 过 $\stackrel{2}{=}=$ స

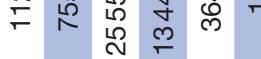

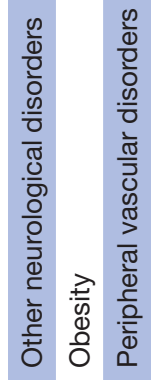

댕ํำ

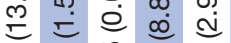

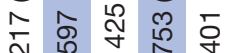
令

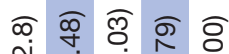
$\cong \pm 0$ @ ㅇำ ம

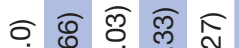
过 巳 巳 ⿺辶寸

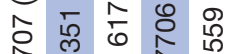

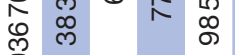
은

กิ ชิ ชิ 过 e e

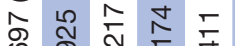
ำ

ช

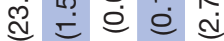
ని న స్ న స

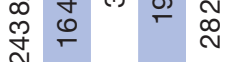

ণิ 융 ฐ ণ

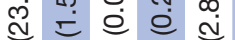

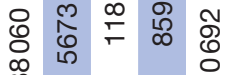

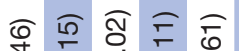
c)

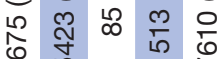
$\stackrel{8}{\forall}$

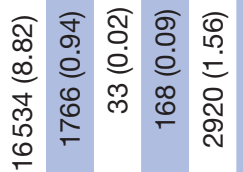

ตั क ळ ก ำ 交 ஏ ซ

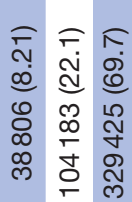

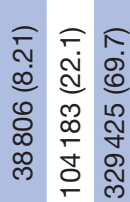

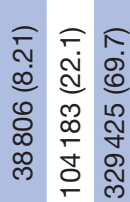

Ф) लै ๙ ले $\infty$ 占

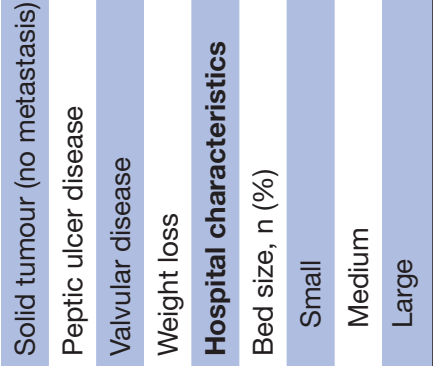




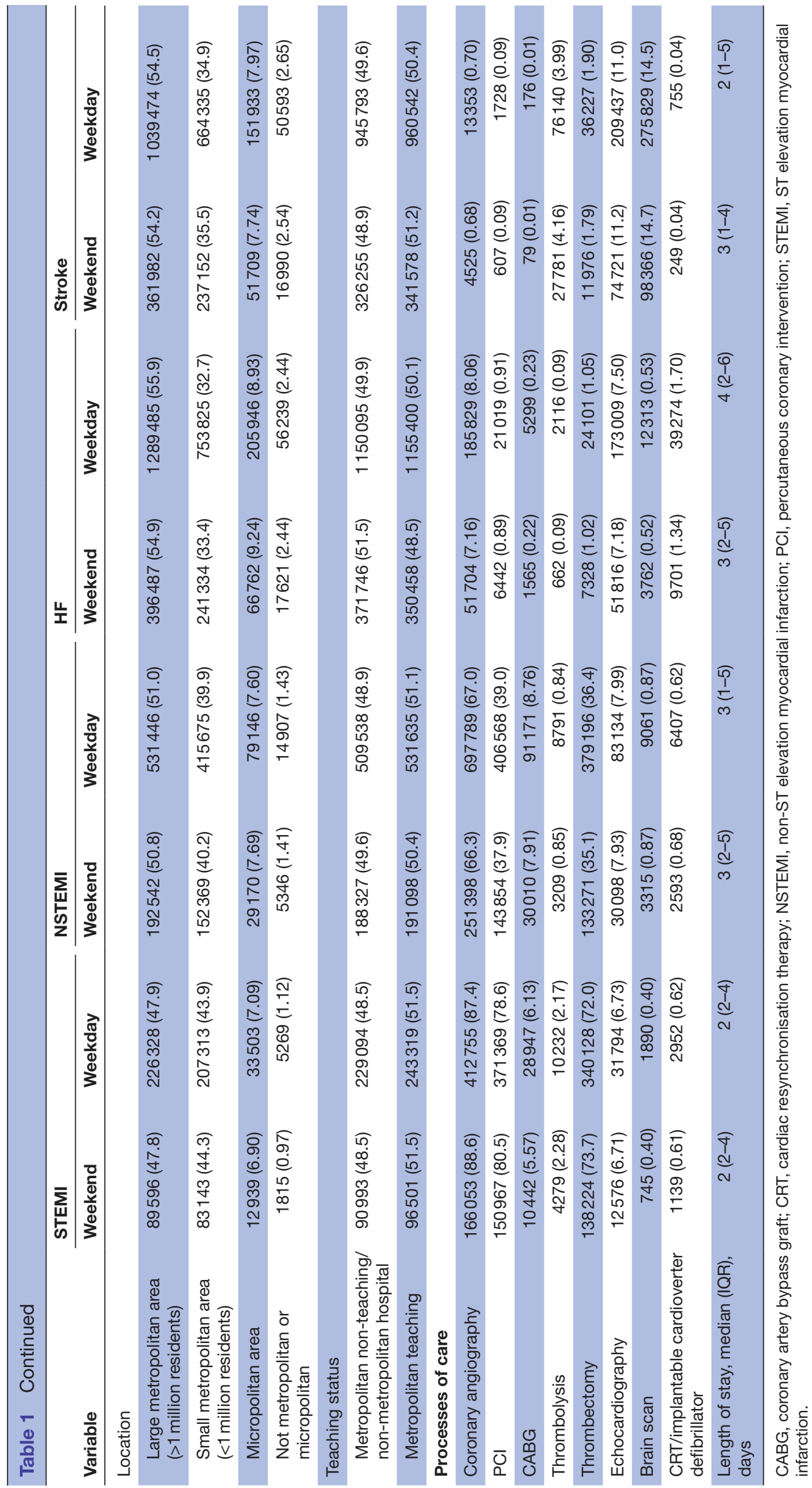


Index admissions for patients aged 18 years or over, with a primary diagnosis of STEMI, NSTEMI, HF or Stroke between 2010 and 2014 (weighted- $n=10,033,539$ )

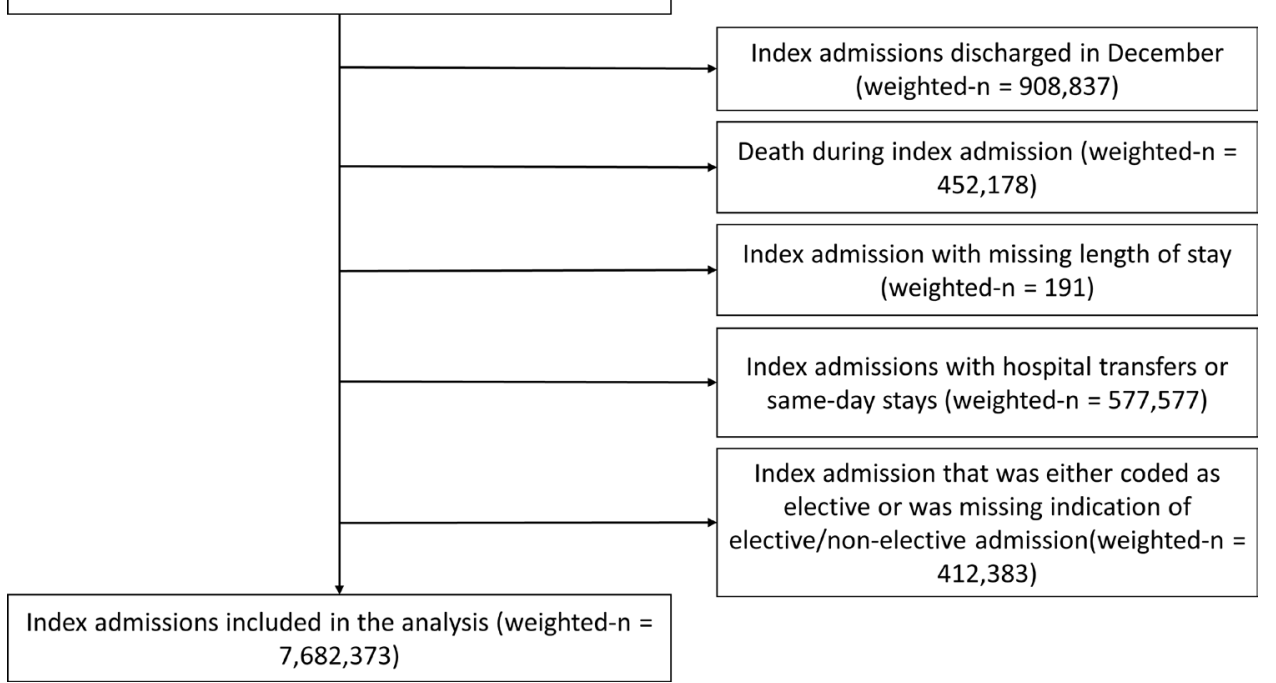

Figure 1 Flow chart of index hospitalisations through the exclusion criteria. HF, heart failure; NSTEMI, non-ST elevation myocardial infarction; STEMI, ST elevation myocardial infarction.

\section{Processes of care}

Observed proportions of each of the in-hospital processes of care are given in table 1 for each of the primary diagnosis groups, with table 3 giving the multivariable adjusted ORs. After multivariable adjustment, STEMI weekend hospitalisations had higher odds of undergoing a coronary angiography, PCI and thrombectomy, but lower odds of CABG compared with a weekday (table 3).

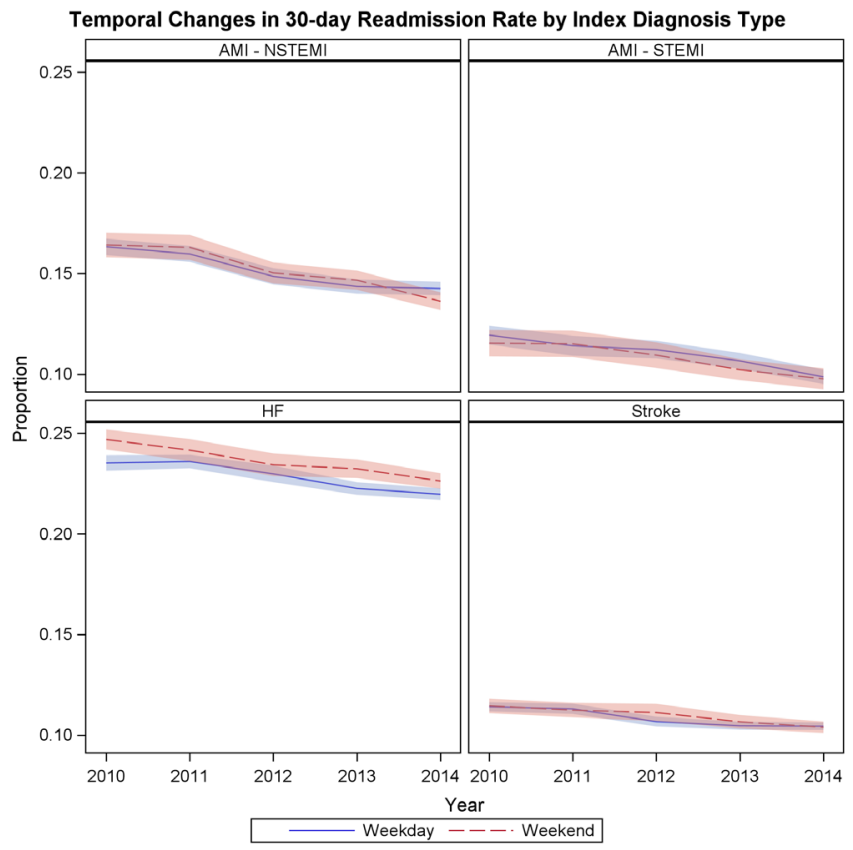

Figure 2 Temporal trends in 30-day readmission by weekend/weekday index hospitalisation, stratified by the category of index diagnosis. AMI, acute myocardial infarction; HF, heart failure; NSTEMI, non-ST elevation myocardial infarction; STEMI, ST elevation myocardial infarction.
In contrast, NSTEMI weekend hospitalisations had lower odds of receiving coronary angiography, PCI, CABG and thrombectomy, compared with weekday hospitalisations. Hospitalisations for $\mathrm{HF}$ at a weekend were associated with lower odds of coronary angiography, echocardiography or CRT/implantable cardioverter-defibrillator compared with weekday HF hospitalisations. Stroke/TIA hospitalisations at a weekend had lower odds of undergoing thrombectomy compared with weekday hospitalisations, but higher odds of thrombolysis, echocardiography and receiving a brain scan. Nevertheless, for all primary diagnosis groups, some of the effect sizes were modest (table 3).

The median LOS for the whole cohort was 3 days (IQR: 2-5) for weekend hospitalisations and 3 days (IQR: 2-5) for the weekday hospitalisations. After multivariable adjustment, weekend hospitalisations for NSTEMI had an increased risk for longer LOS (HR for earlier discharge of $0.961,95 \%$ CI 0.955 to 0.967$)$. Similarly, those admitted at a weekend for a primary diagnosis of stroke/TIA had a longer LOS (HR for earlier discharge of $0.978,95 \%$ CI 0.973 to 0.983 ). In contrast, weekend admissions with HF were associated with increased risk of shorter LOS (HR for earlier discharge of $1.044,95 \%$ CI 1.039 to 1.048$)$, while there was no difference in LOS for STEMI (HR for earlier discharge of $0.997,95 \%$ CI 0.988 to 1.007 ).

\section{DISCUSSION}

To the best of the authors' knowledge, this large study of a national US readmission database is the first to simultaneously analyse the effect of weekend hospitalisation on readmission rates and processes of care for four major cardiovascular diagnoses. Our findings suggest that, in this cohort, the presence of a weekend effect was 
Table 2 Thirty-day readmission numbers and adjusted ORs of unplanned 30-day readmission across weekend versus weekday (reference) index hospitalisation groups

\begin{tabular}{|c|c|c|c|c|c|}
\hline \multirow[b]{2}{*}{$\begin{array}{l}\text { Principle diagnosis within } \\
\text { index hospitalisation }\end{array}$} & \multicolumn{2}{|c|}{ Weekend index hospitalisation } & \multicolumn{2}{|c|}{ Weekday index hospitalisation } & \multirow{2}{*}{$\begin{array}{l}\text { Adjusted OR } \\
(95 \% \mathrm{Cl}) \text { for } \\
\text { weekend index } \\
\text { hospitalisation* }\end{array}$} \\
\hline & $\begin{array}{l}\text { 30-day } \\
\text { readmission, } \\
\text { weighted } n\end{array}$ & $\begin{array}{l}\text { No of } 30 \text {-day } \\
\text { readmission, } \\
\text { weighted } n\end{array}$ & $\begin{array}{l}30 \text {-day } \\
\text { readmission, } \\
\text { weighted } n\end{array}$ & $\begin{array}{l}\text { No of } 30 \text {-day } \\
\text { readmission, } \\
\text { weighted } n\end{array}$ & \\
\hline STEMI & 20256 & 167237 & 52139 & 420274 & $1.010(0.977$ to 1.044$)$ \\
\hline NSTEMI & 57559 & 321867 & 157347 & 883826 & $1.002(0.983$ to 1.021$)$ \\
\hline $\mathrm{HF}$ & 170220 & 551985 & 525888 & 1779606 & 1.045 (1.033 to 1.058$)$ \\
\hline Stroke & 73279 & 594554 & 206932 & 1699403 & 1.014 (0.997 to 1.030$)$ \\
\hline
\end{tabular}

Bold entries indicate significant results. *Adjustment for each of the variables listed in the 'patient characteristics', 'comorbidities' and 'hospital characteristics' sections of table 1.

HF, heart failure; NSTEMI, non-ST elevation myocardial infarction; STEMI, ST elevation myocardial infarction.

relatively modest. Weekend hospitalisations for HF had a statistically significant but clinically modest increase $(4 \%)$ in odds of unplanned 30-day readmission, compared with those admitted during the week. There were differences in several processes of care across weekend and weekday index hospitalisations for STEMI, NSTEMI, HF and stroke/TIA, but some of the effect sizes were modest. Given the large sample size in this study, focusing on effect sizes rather than measures of statistical significance is particularly important.

The potential for disparities in hospital care and outcomes for weekend hospitalisations when staffing

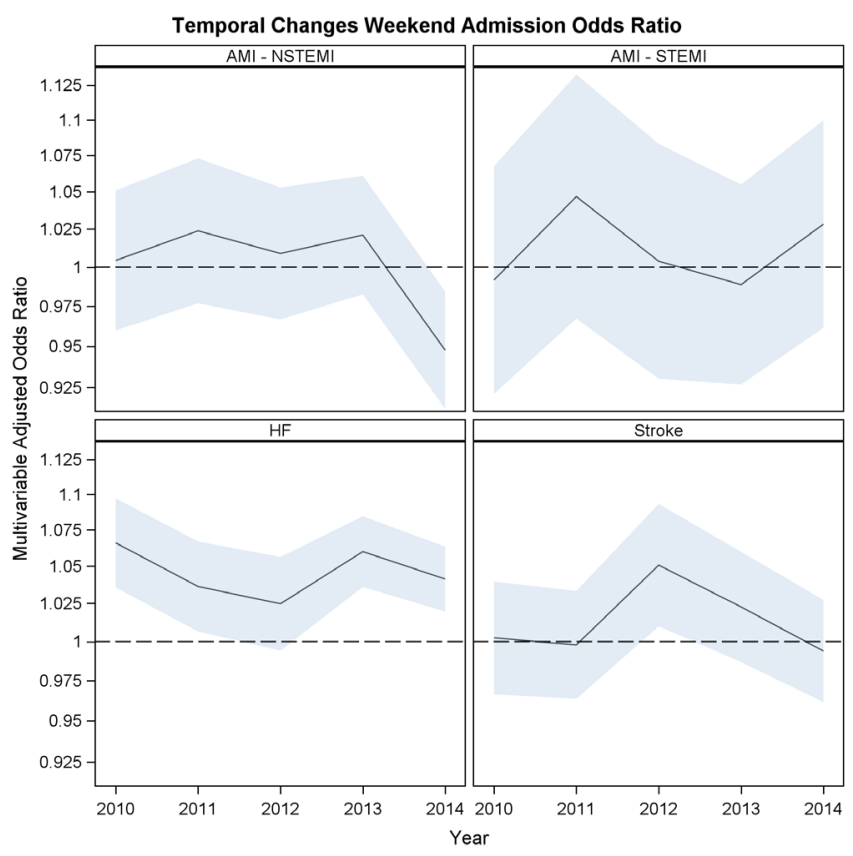

Figure 3 Temporal changes in odds ratios of unplanned 30day readmission across weekend versus weekday (reference) index hospitalisation groups (adjusted for each of the variables listed in the 'patient characteristics', 'comorbidities' and 'hospital characteristics' sections of table 1). AMI, acute myocardial infarction; HF, heart failure; NSTEMI, nonST elevation myocardial infarction; STEMI, ST elevation myocardial infarction. levels and services might be reduced have resulted in a growth of studies exploring the weekend effect. However, the majority of previous studies have focused on short-term outcomes such as in-hospital mortality, with little data on readmission rates. We observed high rates of unplanned 30-day readmission, especially for HF, although there was evidence that these rates have been declining over time. The observed rates and temporal decline of 30-day unplanned readmission are consistent with previous studies ${ }^{30-32}$ and suggests that improvements are being made (eg, through closely monitoring 30-day readmissions or through legislation where unplanned readmissions for HF are non-compensated in the USA). However, even by 2014, the 30-day readmission rates remained significant.

\section{STEMI/NSTEMI index hospitalisations}

There was no statistically significant difference in rates of 30-day readmission between weekend and weekday hospitalisations for STEMI or NSTEMI. This finding suggests that for diagnoses that have widespread emergency service provision (such as PCI for STEMI), guideline-recommended care is delivered irrespective of day of hospitalisation. Hence, the potential of a weekend effect is limited. This is supported by a recent meta-analysis, which found that while there was a marginal increase in the odds of mortality for those admitted for acute coronary syndrome at a weekend, this was not observed in STEMI and NSTEMI subgroups. ${ }^{33}$ Similarly, a study of regional university cardiac centres in the UK demonstrated no differences in mortality rates or processes of care for patients admitted at a weekday or weekend with STEMI or NSTEMI. ${ }^{7}$

However, the current study did find that weekend hospitalisation for STEMI was associated with higher odds of coronary angiography, PCI and thrombectomy. Nonetheless, the effect sizes were small (ORs of 1.063, 1.085 and 1.060 for coronary angiography, PCI and thrombectomy, respectively). In contrast, NSTEMI weekend admissions had lower odds of coronary angiography and PCI. Lower utilisation of invasive cardiac procedures (coronary 
Table 3 Multivariable adjusted ORs of weekend index hospitalisation versus weekday (reference) for processes of care during the index hospitalisation across weekend and weekday hospitalisations

\begin{tabular}{|c|c|c|c|c|}
\hline & STEMI & NSTEMI & HF & Stroke \\
\hline Processes of care & OR $(95 \% \mathrm{Cl})$ * & OR $(95 \% \mathrm{Cl})$ * & OR $(95 \% \mathrm{Cl})$ * & OR $(95 \% \mathrm{Cl})^{*}$ \\
\hline Coronary angiography & $1.063(1.029$ to 1.099$)$ & 0.965 (0.949 to 0.982$)$ & 0.911 (0.893 to 0.929$)$ & 0.965 (0.909 to 1.023$)$ \\
\hline CABG & $0.889(0.851$ to 0.929$)$ & $0.890(0.866$ to 0.914$)$ & 0.989 (0.886 to 1.104$)$ & 1.254 (0.764 to 2.059$)$ \\
\hline Thrombolysis & 1.047 (0.978 to 1.120$)$ & 1.002 (0.927 to 1.084$)$ & 1.032 (0.878 to 1.213$)$ & $1.038(1.011$ to 1.066$)$ \\
\hline Echocardiography & $1.003(0.966$ to 1.041$)$ & $1.000(0.976$ to 1.024$)$ & $0.972(0.955$ to 0.990$)$ & $1.026(1.010$ to 1.043$)$ \\
\hline Brain scan & $1.021(0.893$ to 1.169$)$ & 1.009 (0.949 to 1.073$)$ & 0.995 (0.937 to 1.057$)$ & $1.017(1.003$ to 1.032$)$ \\
\hline $\begin{array}{l}\text { CRT/implantable } \\
\text { cardioverter-defibrillator }\end{array}$ & 0.982 (0.870 to 1.108$)$ & $1.111(1.021$ to 1.208$)$ & $0.812(0.775$ to 0.850$)$ & 0.927 (0.718 to 1.197$)$ \\
\hline
\end{tabular}

Bold entries indicate significant results.

*Adjustment for each of the variables listed in the 'patient characteristics', 'comorbidities', and 'hospital characteristics' sections of table 1. CABG, coronary artery bypass graft; CRT, cardiac resynchronisation therapy; HF, heart failure; NSTEMI, non-ST elevation myocardial infarction; PCI, percutaneous coronary intervention; STEMI, ST elevation myocardial infarction.

angiography, PCI and CABG) for weekend hospitalisations for NSTEMI have been reported previously in the Nationwide Inpatient Sample database, with these data showing a corresponding increase in mortality. ${ }^{6}$ Reassuringly, in the current study, the differences in the rates of angiography between weekend and weekday NSTEMI hospitalisations were small $(0.7 \%$ absolute difference). Examining data on the time between admission (for STEMI or NSTEMI) and the procedure(s), across weekend and weekday subgroups, would be potentially informative, but such data were unavailable in the current study.

\section{HF index hospitalisations}

We observed a statistically significant, but clinically modest, increase in odds of 30-day readmission for $\mathrm{HF}$ hospitalisations at a weekend compared with HF hospitalisations on a weekday. Similar findings were found in a study by Shah et al, who found that Friday discharges for HF had the highest 30-day readmission rate. ${ }^{18}$ In contrast, the Organized Program to Initiate Lifesaving Treatment in Hospitalized Patients With Heart Failure study found no significant differences in mortality or rehospitalisation according to day of hospitalisations for HF. ${ }^{19}$ Our study highlights high unplanned 30-day readmission rates following $\mathrm{HF}$ for both weekend and weekday cohorts (23.6\% for weekend HF index hospitalisations and $22.8 \%$ for weekday HF hospitalisations), which is in line with other studies. ${ }^{182}$ Consequently, while the weekend effect itself seems relatively modest in this study, these high unplanned 30-day readmission rates indicate that there should be a focus on preventative strategies that aim to reduce readmission rates regardless of day of hospitalisation. This is especially important since the majority of readmissions following an index HF hospitalisation were for recurrent $\mathrm{HF}$.

Interestingly, the current study found that some, but not all, indicators of processes of care were reduced for weekend HF hospitalisations. Specifically, HF hospitalisations at a weekend were less likely to have coronary angiography, echocardiography or CRT/implantable cardioverter-defibrillator compared with weekday $\mathrm{HF}$ hospitalisations. The findings of lower odds of echocardiography at a weekend is particularly important since echocardiography is key in identifying causes of cardiac decompensation such as valvular heart disease but is also key in guiding provision of evidence-based therapies based on ejection fraction.

\section{Stroke/TIA index hospitalisations}

The existing evidence of a weekend effect following stroke/TIA hospitalisations is inconsistent. Specifically, while differences in mortality following weekend or weekday hospitalisations for stroke/TIA have been reported, ${ }^{24}{ }^{34}$ other studies have reported no mortality differences. ${ }^{142235}$ Some of these differences might be due to heterogeneity in the organisation of stroke care, ${ }^{34}$ or that stroke outcomes might vary in diurnal patterns rather than simple weekend versus weekday comparisons. ${ }^{22}$ The current study advances the existing evidence base by showing that there was no statistical difference in 30-day readmission rates between weekend hospitalisations for stroke/TIA compared with weekday hospitalisations.

However, there were differences in some procedures for stroke/TIA hospitalisations, with the current study showing that stroke/TIA weekend hospitalisations were more likely to receive thrombolysis, echocardiography or brain scans but had lower odds of receiving thrombectomy. Additionally, stroke/TIA weekend hospitalisations had an increased risk for longer LOS compared with weekday stroke/TIA hospitalisations, which is consistent with previous studies. ${ }^{14} 24$ Our finding that thrombolysis treatment was more likely for weekend stroke/TIA hospitalisations has been previously reported in some, ${ }^{14} 34,36$ but not all, ${ }^{24} 37$ previous studies. It has been suggested 
that weekend hospitalisations present opportunities for quicker arrival at hospital and quicker patient journeys through the healthcare system, thereby increasing the chance of a patient presenting within the 3-hour window for administration of intravenous tissue plasminogen activator. ${ }^{14} 3638$ Additionally, one could hypothesise that demand for diagnostic testing and brain scans could be supported more readily at a weekend due to the reduced demand from elective procedures. Further research is required in this space.

\section{Study limitations}

There are several limitations that should be considered when interpreting the results of this study. First, none of the reported associations can be interpreted as causal due to the possibility of unmeasured confounders and the possibility of unmeasured imbalances in baseline case mix (eg, disease severity or patient behaviour) between weekend and weekday hospitalisation groups. However, the non-elective nature of the diagnoses considered in this study means that the risk of bias induced by possible heterogeneity in disease severity across weekend or weekday groups is arguably low. Second, the use of administrative data to explore the presence of a weekend effect can be challenging due to limitations of coding of acute medical conditions, especially where the reasons for inaccurate coding might differ by day of the week. ${ }^{39}$ To mitigate this, we have followed previous recommendations of excluding all elective hospitalisations and used a stringent selection of ICD-9 codes. ${ }^{39}$ Third, the annualised nature of the NRD means that we were not able to track patients between years, thereby limiting investigation to short-term readmission. Fourth, we could not explore the weekend effect on prescription of recommended drugs/medications since NRD does not record data on pharmacotherapy or prescriptions. Fifth, our analysis could not account for death as a competing risk for readmission since we did not have data on deaths that might have occurred after discharge. Finally, the dataset only included a binary variable indicating if the hospitalisation occurred at a weekend or not; thus, we were not able to explore diurnal variation in outcomes or by individual day of hospitalisation.

\section{CONCLUSION}

In conclusion, this study set out under the hypothesis that 30-day unplanned readmission rates are the same between weekday and weekend hospitalisations for STEMI, NSTEMI, HF and stroke/TIA; we could not find any evidence against this hypothesis. There was a statistically significant difference in some processes of care between weekend and weekday admissions. Unplanned 30-day readmission rates were high regardless of day of index hospitalisation, especially following hospitalisation for HF. Given that unplanned readmissions have both financial consequences and implications for service provision, strategies that aim to reduce readmission rates should be explored, irrespective of day of admission.

\section{Author affiliations}

${ }^{1}$ Division of Informatics, Imaging and Data Science, Faculty of Biology, Medicine and Health, University of Manchester, Manchester Academic Health Science Centre, Manchester, UK

${ }^{2}$ Keele Cardiovascular Research Group, Institute of Primary Care and Health Sciences, Keele University, Stoke-on-Trent, UK

${ }^{3}$ Department of Medicine, McMaster University, Hamilton, Ontario, Canada ${ }^{4}$ Department of Cardiology, Rush University Medical Center, Chicago, Illinois, USA

${ }^{5}$ Department of Medicine (Cardiology), Johns Hopkins University School of Medicine, Baltimore, Maryland, USA

${ }^{6}$ Division of Cardiology, Loma Linda University School of Medicine, Loma Linda, California, USA

${ }^{7}$ Wayne State University, Detroit Medical Center, Detroit, Michigan, USA

${ }^{8}$ University of Pittsburgh Medical Center, Pittsburgh, Pennsylvania, USA

Contributors GPM and MM made substantial contributions to the concept of the work. GPM performed the analysis and drafted the initial version of the manuscript. All named authors helped interpreted the results, revised multiple drafts of the paper critically for important intellectual content and approved the final version of the paper. All authors agree to be accountable for all aspects of the work. The corresponding author (MM) attests that all listed authors meet authorship criteria and that no others meeting the criteria have been omitted.

Funding This research was partially funded by the North Staffordshire Heart Committee.

\section{Competing interests None declared.}

Provenance and peer review Not commissioned; externally peer reviewed.

Data availability statement Data may be obtained from a third party and are not publicly available.

Open access This is an open access article distributed in accordance with the Creative Commons Attribution Non Commercial (CC BY-NC 4.0) license, which permits others to distribute, remix, adapt, build upon this work non-commercially, and license their derivative works on different terms, provided the original work is properly cited, appropriate credit is given, any changes made indicated, and the use is non-commercial. See: http://creativecommons.org/licenses/by-nc/4.0/.

\section{REFERENCES}

1. GBD 2013 Mortality and Causes of Death Collaborators. Global, regional, and national age-sex specific all-cause and causespecific mortality for 240 causes of death, 1990-2013: a systematic analysis for the global burden of disease study 2013. Lancet 2015;385:117-71.

2. Bhatnagar $\mathrm{P}$, Wickramasinghe $\mathrm{K}$, Williams J, et al. The epidemiology of cardiovascular disease in the UK 2014. Heart 2015;101:1182-9.

3. Schmulewitz L, Proudfoot A, Bell D. The impact of weekends on outcome for emergency patients. Clin Med 2005;5:621-5.

4. Bell CM, Redelmeier DA. Mortality among patients admitted to hospitals on weekends as compared with weekdays. N Engl J Med 2001;345:663-8.

5. Sorita A, Lennon RJ, Haydour Q, et al. Off-hour admission and outcomes for patients with acute myocardial infarction undergoing percutaneous coronary interventions. Am Heart J 2015;169:62-8.

6. Khoshchehreh M, Groves EM, Tehrani D, et al. Changes in mortality on weekend versus weekday admissions for acute coronary syndrome in the United States over the past decade. Int J Cardiol 2016;210:164-72.

7. Martin GP, Kinnaird T, Sperrin M, et al. Effect of weekend admission on process of care and clinical outcomes for the management of acute coronary syndromes: a retrospective analysis of three UK centres. BMJ Open 2017;7:e016866.

8. McNamara RL, Wang Y, Herrin J, et al. Effect of door-to-balloon time on mortality in patients with ST-segment elevation myocardial infarction. J Am Coll Cardiol 2006;47:2180-6.

9. O'Gara PT, Kushner FG, Ascheim DD, et al. 2013 ACCF/AHA guideline for the management of ST-elevation myocardial infarction. $J$ Am Coll Cardiol 2013;61:e78-140.

10. Amsterdam EA, Wenger NK, Brindis RG, et al. 2014 AHA Aacc guideline for the management of patients with non-ST-elevation 
acute coronary syndromes: a report of the American College of Cardiology/American heart association Task force on practice guidelines. J Am Coll Cardiol 2014;64:e139-228

11. Roffi M, Patrono C, Collet J-P, et al. 2015 ESC guidelines for the management of acute coronary syndromes in patients presenting without persistent ST-segment elevation. Eur Heart $J$ 2016;37:267-315.

12. Gallagher SM, Lovell MJ, Jones DA, et al. Does a 'direct' transfer protocol reduce time to coronary angiography for patients with nonST-elevation acute coronary syndromes? A prospective observational study. BMJ Open 2014;4:e005525.

13. Angus DC, Shorr AF, White A, et al. Critical care delivery in the United States: distribution of services and compliance with Leapfrog recommendations*. Crit Care Med 2006;34:1016-24.

14. Hoh BL, Chi Y-Y, Waters MF et al. Effect of weekend compared with weekday stroke admission on thrombolytic use, in-hospital mortality, discharge disposition, hospital charges, and length of stay in the nationwide inpatient sample database, 2002 to 2007. Stroke 2010;41:2323-8.

15. Kostis WJ, Demissie K, Marcella SW, et al. Weekend versus weekday admission and mortality from myocardial infarction. $N$ Engl $J$ Med 2007;356:1099-109.

16. Sorita A, Ahmed A, Starr SR, et al. Off-hour presentation and outcomes in patients with acute myocardial infarction: systematic review and meta-analysis. BMJ 2014;348:f7393.

17. Berger A, Stauffer J-C, Radovanovic D, et al. Comparison of inhospital mortality for acute myocardial infarction in Switzerland with admission during routine duty hours versus admission during out of hours (insight into the AmiS plus registry). Am J Cardiol 2008;101:422-7.

18. Shah M, Patnaik S, Patel B, et al. The day of the week and acute heart failure admissions: relationship with acute myocardial infarction, 30-day readmission rate and in-hospital mortality. Int $J$ Cardiol 2017;249:292-300.

19. Fonarow GC, Abraham WT, Albert NM, et al. Day of admission and clinical outcomes for patients hospitalized for heart failure: findings from the organized program to initiate lifesaving treatment in hospitalized patients with heart failure (OPTIMIZE-HF). Circ Heart Fail 2008;1:50-7.

20. Horwich TB, Hernandez AF, Liang L, et al. Weekend hospital admission and discharge for heart failure: association with quality of care and clinical outcomes. Am Heart J 2009;158:451-8.

21. Hamaguchi S, Kinugawa S, Tsuchihashi-Makaya M, et al. Weekend versus weekday hospital admission and outcomes during hospitalization for patients due to worsening heart failure: a report from Japanese cardiac registry of heart failure in cardiology (JCARECARD). Heart Vessels 2014;29:328-35.

22. Bray BD, Cloud GC, James MA, et al. Weekly variation in healthcare quality by day and time of admission: a nationwide, registrybased, prospective cohort study of acute stroke care. Lancet 2016;388:170-7

23. Adil MM, Vidal G, Beslow LA. Weekend effect in children with stroke in the nationwide inpatient sample. Stroke 2016;47:1436-43.
24. Turner M, Barber M, Dodds $\mathrm{H}$, et al. Stroke patients admitted within normal working hours are more likely to achieve process standards and to have better outcomes. J Neurol Neurosurg Psychiatry 2016;87:138-43.

25. HCUP. 2013 introduction to the NRD. Rockville, MD: Agency for Healthcare Research \& Quality, 2015.

26. Blecker S, Paul M, Taksler G, et al. Heart Failure-Associated hospitalizations in the United States. J Am Coll Cardiol 2013;61:1259-67.

27. Leone MA, Capponi A, Varrasi C, et al. Accuracy of the ICD-9 codes for identifying TIA and stroke in an Italian automated database. Neurol Sci 2004;25:281-8.

28. Kwok CS, Rao SV, Gilchrist I, et al. Relation between age and unplanned readmissions after percutaneous coronary intervention (findings from the nationwide readmission database). Am J Cardiol 2018;122:220-8.

29. Rothendler JA, Rose AJ, Reisman JI, et al. Choices in the use of ICD-9 codes to identify stroke risk factors can affect the apparent population-level risk factor prevalence and distribution of CHADS2 scores. Am J Cardiovasc Dis 2012;2:184-91.

30. Dunlay SM, Weston SA, Killian JM, et al. Thirty-Day rehospitalizations after acute myocardial infarction. Ann Intern Med 2012;157:11-18.

31. Kim LK, Yeo I, Cheung JW, et al. Thirty-Day readmission rates, timing, causes, and costs after ST-Segment-Elevation myocardial infarction in the United States: a national readmission database analysis 2010-2014. J Am Heart Assoc 2018;7.

32. Dharmarajan K, Hsieh AF, Lin Z, et al. Diagnoses and timing of 30-day readmissions after hospitalization for heart failure, acute myocardial infarction, or pneumonia. JAMA 2013;309:355-63.

33. Kwok CS, Al-Dokheal M, Aldaham S, et al. Weekend effect in acute coronary syndrome: a meta-analysis of observational studies. Eur Hear J Acute Cardiovasc Care 2018.

34. McKinney JS, Deng Y, Kasner SE, et al. Comprehensive stroke centers overcome the weekend versus weekday gap in stroke treatment and mortality. Stroke 2011;42:2403-9.

35. Albright KC, Savitz SI, Raman R, et al. Comprehensive stroke centers and the 'Weekend Effect': The SPOTRIAS experience on behalf of the SPOTRIAS investigators. Cerebrovasc Dis 2012;34:424-9.

36. Kazley AS, Hillman DG, Johnston KC, et al. Hospital care for patients experiencing weekend vs weekday stroke. Arch Neurol 2010;67:39-44

37. Lees KR, Ford GA, Muir KW, et al. Thrombolytic therapy for acute stroke in the United Kingdom: experience from the safe implementation of thrombolysis in stroke (SITS) register. QJM 2008;101:863-9.

38. Fisher M, Ringleb PA, Schellinger PD, et al. Thrombolytic therapy within 3 to 6 hours after onset of ischemic stroke: useful or harmful? Stroke 2002;33:1437-41.

39. Li L, Rothwell PM. Biases in detection of apparent "weekend effect" on outcome with administrative coding data: population based study of stroke. BMJ 2016;353. 